\title{
Modelo empresarial de alianza público- privada a través de los municipios para impulsar el desarrollo socioeconómico
}

\author{
Business model of a private-public alliance through \\ local governments to promote socio-economic \\ development
}

José Kennedy Ollague Valarezo

Universidad Técnica de Machala

(Machala - Ecuador)

jollague@utmachala.edu.ec

Revista Cumbres Vol.3 №2

Versión impresa ISSN 1390-9541

Versión electrónica ISSN 1390-3365

http://investigacion.utmachala.edu.ec/revistas/index.php/Cumbres 


\section{RESUMEN}

Con el objetivo de proponer un modelo empresarial mediante alianzas público-privada (APP), a través de los municipios para el desarrollo socioeconómico de la provincia de El Oro, Ecuador, se desarrolló una investigación de tipo no experimental, donde se encuestaron 440 líderes de opinión, profesores universitarios y personas de amplios conocimientos relacionados con las alianzas público-privadas en la provincia de El Oro. Además, se efectuaron entrevistas a alcaldes, concejales y profesionales del ramo. La asociación entre las variables independientes y dependientes identificadas se determinó mediante el Coeficiente Rho de Spearman y una vez comprobada su correlación se aplicó el Análisis de Componentes Principales que permitió reducir la dimensionalidad del conjunto de variables. El contraste de hipótesis se efectuó mediante la prueba chi-cuadrado de independencia y la veracidad de los datos obtenidos se realizó mediante análisis de confiabilidad, determinándose el índice Alfa de Cronbach el cual es un indicador de consistencia interna del modelo. El procesamiento de los datos se efectuó mediante el paquete estadístico SPSS versión 22 de prueba para Windows. Se diseñó un modelo empresarial basado en alianzas estratégicas interinstitucionales que permitan la implementación de proyectos de inversión con democratización de la propiedad. Se concluye que es factible la implementación de un modelo de empresa estándar capaz de generar prosperidad local basándose en el desarrollo de las potencialidades que tiene cada jurisdicción, sea esta un municipio o cantón, departamento, región o provincia, por lo que su aplicabilidad es de carácter universal.

Palabras clave: Asociación público-privada, municipalidades, modelo empresarial, alianzas estratégicas, democratización de capital, desarrollo socioeconómico.

\section{ABSTRACT}

The objective of this study was to propose a business model based on public-private partnership (PPP), through municipalities for the socioeconomic development of El Oro province, Ecuador. A non-experimental type of research was applied; 440 opinion leaders, professors and people who have an extensive knowledge of public-private partnerships in the El Oro were surveyed. In addition, interviews with mayors, city councilors, and professionals in the field were also conducted. The association between the independent and dependent variables identified was determined by Spearman Rho Ratio. Once their correlation was verified, the Analysis of Principal Components was applied. It allowed reducing the dimensionality of the set of variables through the creation of new factors. The contrast of hypothesis was done using the chi-square test of independence and the accuracy of the data was obtained by reliability analysis. The Cronbach Alpha rate was used to evaluate the internal consistency of the proposed model. Data processing was performed using the SPSS Statistical Package 22 trial version for Windows. An

\section{Cumbres}


enterprise model was designed based on strategic inter-institutional alliances that allow the implementation of investment projects with the democratization of property. It is concluded that it is feasible to implement a standard enterprise model capable of generating local prosperity based on the development of the potentialities of each jurisdiction, either as a municipality or canton, department, region or province. This means that this model can be used in different areas.

Keywords: Public-private partnerships, municipalities, business model, strategic alliances, capital democratization, socio-economic development.

\section{INTRODUCCIÓN}

Las asociaciones entre lo público y privado han estado presente por mucho tiempo en el mundo, apoyaron la exploración y desarrollo del comercio occidental con el Nuevo Mundo y el Lejano Oriente, las que permitieron el desarrollo de la infraestructura con la que cuenta el nuevo mundo (Sepúlveda, 2014). Se podría decir por tanto que el sector público históricamente se ha esmerado en lograr acuerdos con el sector privado para alcanzar los objetivos de beneficiar a sus ciudadanos. La asociatividad entre el sector público y privado es diferente en la actualidad, puesto que la concepción moderna de asociación público-privada, comprende la búsqueda de acuerdos de cooperación para temas específicos (Moore, 2006)

Generalmente las estrategias no son de instauración exclusiva del gobierno central, sino que nacen de alianzas público-privadas teniendo así: la participación de la sociedad civil, elementos de orientación política y creación de consenso. Este suceso, ha adoptado diversas representaciones en los distintos países que se ha incluido en esta metodología. Si bien, el éxito de una estrategia de ese tipo depende de factores políticos y del diseño técnico-financiero, es importante la existencia de un marco legal apropiado que pueda aplicarse, como en Ecuador, la Ley Orgánica de Incentivos para Asociaciones Público-Privadas y la Inversión Extranjera, creada en diciembre de 2015.

Existen organizaciones que han ido cambiando su rol en la administración pública como el caso de las municipalidades (Sierra \& Padilla, 1997), que han evolucionado de estructuras cerradas a modernos esquemas con amplia apertura para intervenir en procesos productivos inclusive, dentro de su jurisdicción. Las municipalidades se constituyen en las entidades convocadas a generar desarrollo local con impacto nacional e internacional y son las protagonistas del presente modelo, como menciona Bernal, Pumar \& Zelada (2005), municipio productivo es aquel que orienta la inversión privada conforme las señales del mercado motivando el desarrollo de actividades económicas sostenibles y equitativas. Además de este promotor (principal), otras organizaciones que actualmente tienen un rol similar son las Instituciones de Educación Superior (IES), que cada vez van tomando parte en el desarrollo, con actividades de investigación y vinculación con la sociedad, buscando, al igual que las municipalidades, el bienestar general de la población. Es- 
tas organizaciones eventualmente aunadas a otras entidades especializadas como ministerios, ONG y fundaciones, pueden constituir equipos capaces de encontrar oportunidades para el desarrollo local sostenible.

La integración inducida por las municipalidades con las IES y otras entidades mediante el establecimiento de alianzas estratégicas interinstitucionales, pueden resultar en entes impulsadores capaces de promover el desarrollo local usando como medio el esquema de la asociación entre el sector público y privado. Las asociaciones público-privadas (APP), van más allá de una simple relación, son un medio de colaboración entre estos sectores con el propósito de lograr el máximo bienestar social (Vasallo \& Izquierdo, 2010). Estas APP se constituyen bajo un modelo empresarial que permite generar empleo, pero, sobre todo, democratizar el capital y por tanto la propiedad de los medios de producción y responden a la necesidad de aprovechar los recursos propios de cada Municipio considerado como potencialidades. Un modelo empresarial social e inclusivo financiado a través de las Bolsas de Valores, permitiendo la transferencia de los que disponen de excedentes hacia los que necesitan de ellos (Bolsa de valores de Quito, 2016), obtenidos por intermedio de instrumentos financieros de renta fija (bonos) y variable (acciones) (H. Congreso Nacional, 2008).

En esta investigación no se pretende demostrar la existencia de una relación causal, sino hacer hincapié en la eficiencia de todos los aspectos, centrándose en la coherencia y la eficacia de las metas establecidas, flexibilidad, corrección de errores, y la posibilidad de que la aplicación de una propuesta de modelos de APP no esté dependiente a los ciclos políticos. El objetivo del trabajo consiste en proponer un modelo empresarial mediante alianzas APP, a través de los municipios para el desarrollo socioeconómico de la provincia de El Oro.

\section{MATERIALES Y MÉTODOS}

La investigación es de tipo no experimental, basada en un diseño transeccional, descriptivo y correlacional (Hernández, Fernández, \& Baptista, 2014) en el cual la unidad de análisis estuvo constituida por el personal que labora en las municipalidades, cámaras de comercio y producción, universidades y población en general de la provincia de El Oro. El tamaño de la muestra fue de 440 personas, la misma se determinó a partir de la aplicación de la fórmula para poblaciones finitas (Rodríguez, Gil, \& García, 1996) y se tomó como punto de partida los 680.645 habitantes de la provincia (INEC, 2016). Para la selección de la muestra se aplicó el tipo de muestreo probabilístico donde cada individuo tiene la misma posibilidad de ser elegido.

La técnica utilizada para la recolección de datos fue la encuesta de opinión, la cual fue desarrollada a través de un instrumento (cuestionario con 19 ítems) previamente elaborado y aplicado en diferentes contextos relacionados con la actividad de las empresas público-privadas de la provincia de El Oro (Hernández, Fernández, \& Baptista, 2014). Las respuestas fueron es-

\section{Cumbres}


tructuradas con la utilización de escalas tipo Likert (Medina, Piloto, \& Nogueira, 2011) dándole valores de uno a cinco, distribuidas en totalmente de acuerdo (5), de acuerdo (4), indiferente (3), en desacuerdo (2) y totalmente en desacuerdo (1).

Se identificó como variable independiente (X) el modelo empresarial de alianza público privado (APP), dentro del cual se incluyeron como indicadores la empresa, los actores del modelo y la asociación del sector público con el privado, y como variable dependiente (Y) se identificó el desarrollo socioeconómico de la provincia de El Oro, dentro del que se encuentran los indicadores de desarrollo económico y social (Ríos \& Torres, 2013).

La asociación entre las variables identificadas (categóricas ordinales) se determinó mediante el Coeficiente de correlación Rho de Speerman. Una vez comprobada la correlación entre las variables analizadas y con el objetivo de condensar la información contenida en las variables originales (ítems del instrumento aplicado) en un conjunto más pequeño de variables (factores) con una mínima pérdida de la información se utilizó el Análisis de Componentes Principales (ACP) (Hair, Anderson, Tatham, \& Black, 1999), el cual ordena cada componente de forma decreciente por el valor de su varianza, lo que posibilita explicar el fenómeno estudiado en los primeros Componente Principales creados reduciéndose su dimensionalidad. Si el coeficiente de Káiser-Meyer-Olkin (KMO) es mayor a 0,5 es un indicador de que la aplicación del ACP es adecuada y mientras más se acerca a 1 es mejor el procedimiento escogido y si en la Prueba de Esfericidad de Bartlett se obtiene un valor menor que el error permitido $(0,05)$ se confirma que la utilización del ACP es correcta (Jolliffe, 1986; Almenara, Gonzalez, Garcia, \& Peña, 1998).

El contraste de las hipótesis se realizó mediante la prueba chi-cuadrado de independencia (Pearson, 1900) a partir de la comparación de las variables de mayor peso dentro de los componentes principales formados para las variables independientes y dependientes.

Con la finalidad de determinar la veracidad del modelo propuesto se utilizó la información proveniente de las encuestas, dentro de las que se escogió a 54 líderes de opinión (profesores universitarios, alcaldes, concejales, directores o gerentes de las empresas municipales, presidentes de las cámaras de comercio y de la producción de la provincia de El Oro seleccionados por su trayectoria y experiencia en el área empresarial) de los diferentes cantones de la provincia (considerados para este caso como grupo de expertos) y teniendo en cuenta los ítems relacionados con la propuesta del modelo EMPAPP, se procedió a realizar un análisis de confiabilidad (González, 2008) y se determinó el índice Alfa de Cronbach (Cronbach, 1951). El análisis de los datos se desarrolló mediante la utilización del Paquete Estadístico SPSS versión 22 de prueba para Windows (IBM, 2013).

\section{RESULTADOS Y DISCUSIÓN}

La provincia de El Oro, como jurisdicción forma parte de la Región Litoral, posee una extensión de $6.188 \mathrm{~km}^{2}$, Machala constituye su capital y la 
quinta ciudad más poblada e importante desde el punto de vista económico en el país, siendo el segundo puerto marítimo después de Guayaquil. Es un territorio agrícola-productivo y con gran movimiento comercial, constituyéndose en el polo económico del sur ecuatoriano. Sus pobladores se dedican principalmente a la actividad bananera, por ello es reconocida internacionalmente como "Provincia Bananera del Ecuador" (Neira, 2014).

Previo al análisis estadístico y teniendo en cuenta lo descrito por Ríos y Torres (2013) se efectuó la clasificación de variables en independientes (VI) y dependientes (VD) (Tabla 1).

La interacción entre las variables independientes y dependientes analizadas fue determinada mediante el Coeficiente de Correlación Rho de Spearman (r) el cual muestra que se presenta asociación positiva entre todas las variables y los valores de correlación marcados en negrita que indican una asociación moderada, buena o muy buena entre las variables analizadas

Tabla 1. Identificación de las variables objeto de estudio a partir de su denominación

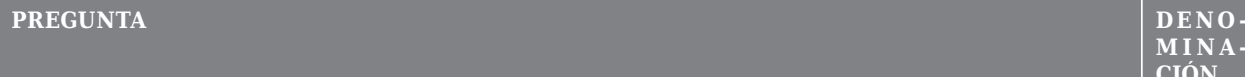

Variables independientes (X)

¿Estaría de acuerdo en la creación de un Departamento de Emprendimiento Municipal X1 encargado de orientar y promover la inversión en los municipios de la provincia de El Oro?

¿Considera usted que se deberían establecer alianzas estratégicas entre las municipa- X2 lidades con universidades y otras entidades, para conformar equipos de investigación multidisciplinarios para la búsqueda de oportunidades de negocios en los municipios de la provincia de El Oro?

¿Cree usted que la inversión privada podría dirigirse hacia la oferta de bienes y servicios X3 a la comunidad en los municipios de la provincia de El Oro?

¿Considera razonable la asociación entre el sector público y privado para la búsqueda de X4 oportunidades de negocios en los municipios de la provincia de El Oro?

¿Estaría de acuerdo en que las oportunidades de negocios se conviertan en compañías X5 de inversión público-privada en los municipios de la provincia de El Oro?

¿Estaría dispuesto a pagar por los servicios o bienes que oferte la inversión público-pri- X6 vada, a través de los municipios de la provincia de El Oro?

¿Estaría de acuerdo que las compañías de inversión público-privada deban administrar- X7 se mediante Gobiernos corporativos?

¿Estaría de acuerdo que los integrantes del equipo multidisciplinario laboren en las X8 compañías de inversión público-privada en los municipios de la provincia de El Oro?

¿Estaría de acuerdo en que sea la Bolsa de Valores el medio por el que se obtengan los X9

fondos para estas compañías de inversión público-privada de los municipios de la provincia de El Oro?

¿Considera usted que se deba permitir a la ciudadanía invertir para posibilitar la democratización de la propiedad en empresas de inversión público-privada creadas por los municipios de la provincia de El Oro?

¿Considera usted que deba abrirse la posibilidad a la inversión extranjera en las compañías de inversión público-privada promovidas por los municipios de la provincia de El Oro?

¿Estaría de acuerdo en que debe existir un límite para los inversionistas procurando democratizar el capital social en las compañías de inversión público-privada en los municipios de la provincia de El Oro?

¿Considera adecuado que los proyectos seleccionados como oportunidades de negocios sean sometidos a evaluación externa para garantizar la fiabilidad y transparencia del estudio y constituirse en empresas público-privada en los municipios de la provincia de El Oro?

\section{Cumbres}


Tabla 1. Identificación de las variables objeto de estudio a partir de su denominación

$$
\text { Variables dependientes }(\mathrm{Y})
$$

¿Considera usted que las empresas de inversión público-privada creadas por las munici- Y1 palidades favorecerán el desarrollo económico en los cantones de la provincia de El Oro?

¿Considera usted que la inversión público-privada, a través de los municipios, contribui- Y2 rá a la generación de empleos en los cantones de la provincia de El Oro?

¿Considera usted que la inversión público-privada, a través de los municipios, contribui- Y3 rá al mejoramiento de los indicadores sociales en los cantones de la provincia de El Oro?

¿Considera usted que la inversión público-privada, a través de los municipios contribui- $\quad$ Y4 rá a disminuir los niveles de pobreza y desigualdad en los cantones de la provincia de El Oro?

¿Considera usted que la inversión público-privada, a través de los municipios contribuirá Y5 al desarrollo de estudios de impacto ambiental en los cantones de la provincia de El Oro?

¿Considera usted que la inversión público-privada a través de los municipios favorecerá Y6 la implementación de programas de tratamiento y aprovechamiento de residuos en los cantones de la provincia de El Oro?

y que para todos los casos son significativas para un alfa $<0,01$; por lo que desde el punto de vista estadístico es correcto realizar un ACP.

El coeficiente KMO obtenido en el ACP realizado para las variables independientes $(0,863)$ al ser superior a 0,5 es un indicador de que las variables introducidas se encuentran altamente correlacionadas entre ellas, lo que se confirma con el valor de significación alcanzado en la Prueba de Esfericidad de Bartlett (significación=0,000), evidenciándose que se puede reducir la dimensionalidad de las variables y que es posible la creación de nuevos factores o componentes principales incorrelados entre sí. De acuerdo al análisis factorial realizado para las variables independientes se forman tres componentes principales que explican un $65,72 \%$ de la variación total explicada para estas variables (Tabla 2).

Tabla 2. Varianza total explicada para los componentes principales formados para variables independientes

\begin{tabular}{|c|c|c|c|c|c|c|}
\hline \multirow[t]{2}{*}{$\begin{array}{l}\text { C O M P O - } \\
\text { NENTE }\end{array}$} & \multicolumn{3}{|c|}{ AUTOVALORES INICIALES } & \multicolumn{3}{|c|}{$\begin{array}{l}\text { SUMAS DE EXTRACCIÓN DE CARGAS } \\
\text { AL CUADRADO }\end{array}$} \\
\hline & Total & $\begin{array}{l}\text { \% de va- } \\
\text { rianza }\end{array}$ & $\begin{array}{l}\% \text { acumu- } \\
\text { lado }\end{array}$ & Total & $\begin{array}{l}\text { \% de va- } \\
\text { rianza }\end{array}$ & $\begin{array}{l}\% \text { acumu- } \\
\text { lado }\end{array}$ \\
\hline 1 & 5,723 & 44,027 & 44,027 & 5,723 & 44,027 & 44,027 \\
\hline 2 & 1,726 & 13,280 & 57,306 & 1,726 & 13,280 & 57,306 \\
\hline 3 & 1,094 & 8,414 & 65,720 & 1,094 & 8,414 & 65,720 \\
\hline
\end{tabular}

De acuerdo al análisis factorial, para las variables independientes, en la matriz de componentes rotados se destacan siete variables, la X1, X5, X9, $\mathrm{X} 12, \mathrm{X} 2, \mathrm{X} 6$ y X10 y las de mayor peso dentro de los tres componentes creados son la X5 (creación de compañías de inversión público-privada en los municipios) con un valor de 0,910, la X9 (bolsa de valores como medio de financiamiento) que alcanza un valor de 0,902, la X1 (creación de Unidades Administrativas en las municipalidades) que obtiene una cifra de 0,897, la X12 (límite para los inversionistas) con 0,699, la X2 (alianzas estratégicas interinstitucionales) que alcanza 0,892, la X6 (pago de bienes y servicios 
producto de las APP) que llega a 0,891 y la X10 (inversión de ciudadanos comunes para democratización del capital) que alcanza un valor de 0,890. El gráfico de sedimentación representa los autovalores de los diferentes componentes principales formados para las variables independientes y donde se confirma que los componentes escogidos son los tres primeros al presentar auto valores mayores a 1 (Figura 1)

De acuerdo al análisis factorial, para las variables independientes, en la matriz de componente rotado se destacan siete variables que podrían ser $\mathrm{X} 1, \mathrm{X} 5$, $\mathrm{X} 9, \mathrm{X} 12, \mathrm{X} 2, \mathrm{X} 6$ y X10. Por lo tanto, el análisis factorial indica que las variables independientes de mayor peso dentro de los tres componentes creados son la X5 (creación de compañías de inversión público-privada en los municipios) con un valor de 0,910, la X9 (bolsa de valores como medio de financiamiento) que alcanza un valor de 0,902, la X1

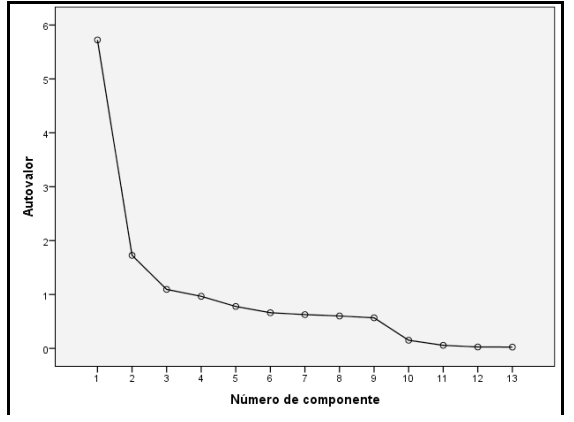

Figura 1. Gráfico de sedimentación que representa los autovalores de los diferentes componentes principales formados para las variables independientes (creación de Unidades Administrativas en las municipalidades) que obtiene una cifra de 0,897, la X12 (límite para los inversionistas) con 0,699 , la X2 (alianzas estratégicas interinstitucionales) que alcanza 0,892, la X6 (pago de bienes y servicios producto de las APP) que llega a 0,891 y la X10 (inversión de ciudadanos comunes para democratización del capital) que alcanza un valor de 0,890 (Tabla 3).

Tabla 3. Matriz de componente rotado según el análisis de componentes principales aplicado a las variables independientes

\begin{tabular}{|c|c|c|c|}
\hline \multirow{2}{*}{ VARIABLE INDEPENDIENTE } & \multicolumn{3}{|c|}{ COMPONENTE } \\
\hline & 1 & 2 & 3 \\
\hline $\mathrm{X} 1$. & 0,897 &, 241 & ,309 \\
\hline X2. & 0,336 & ,231 & ,892 \\
\hline X3. & 0,139 &, 551 & ,083 \\
\hline $\mathrm{X} 4$. & $-0,004$ &, 550 & ,245 \\
\hline X5. & 0,910 & ,208 & ,307 \\
\hline X6. & 0,281 &, 145 & ,891 \\
\hline X7. & 0,148 &, 586 &, 023 \\
\hline X8. & 0,178 &, 590 & ,093 \\
\hline X9. & 0,902 & ,228 & ,324 \\
\hline $\mathrm{X} 10$. & 0,320 & ,214 & ,890 \\
\hline $\mathrm{X} 11$. & 0,176 & 625 & ,109 \\
\hline $\mathrm{X} 12$. & 0,152 & ,699 & ,060 \\
\hline X13. & 0,035 &, 635 & ,231 \\
\hline
\end{tabular}

Método de extracción: ACP. Método de rotación: Varimax con normalización Káiser. 
Para el caso de las variables dependientes el coeficiente KMO obtenido en el ACP realizado $(0,842)$ muestra que las variables introducidas se encuentran altamente correlacionadas entre ellas, confirmándose con el valor de significación alcanzado en la Prueba de esfericidad de Bartlett (sig.=0,000), que se puede reducir la dimensionalidad de las variables y crear nuevos factores o componentes principales incorrelados entre sí. El análisis factorial realizado evidencia la formación de dos componentes principales que explican un 84,52\% de la variación total explicada para estas variables (Tabla 4).

Tabla 4. Varianza total explicada para los componentes principales formados para las variables dependientes

\begin{tabular}{|l|l|l|l|l|l|l|l|}
\hline COMPONENTE & \multicolumn{4}{|l|}{ AUTOVALORES INICIALES } & \multicolumn{3}{l|}{$\begin{array}{l}\text { SUMAS DE EXTRACCIÓN DE CAR- } \\
\text { GAS AL CUADRADO }\end{array}$} \\
\hline & Total & $\begin{array}{l}\% \text { de } \\
\text { rianza }\end{array}$ & $\begin{array}{l}\% \text { vacumu- } \\
\text { lado }\end{array}$ & Total & $\begin{array}{l}\% \text { de } \\
\text { rianza- }\end{array}$ & $\begin{array}{l}\text { vacu- } \\
\text { mulado }\end{array}$ \\
\hline 1 & 3,981 & 66,343 & 66,343 & 3,981 & 66,343 & 66,343 \\
\hline 2 & 1,091 & 18,180 & 84,523 & 1,091 & 18,180 & 84,523 \\
\hline
\end{tabular}

De acuerdo al análisis factorial, para las variables dependientes, en la matriz de componentes rotados se destacan por su orden seis variables Y1, Y3, Y5, Y4, Y6 y Y2, y las VD de mayor peso dentro de los dos componentes creados son la Y1 (desarrollo económico), Y3 (mejoramiento de indicadores sociales), Y5 (desarrollo con estudios de impacto ambiental), Y4 (disminución de niveles de pobreza y desigualdad), Y6 (tratamiento y aprovechamiento de residuos) y la Y2 (generación de empleos).

El gráfico de componentes principales en espacio rotado para las variables dependientes muestra los nuevos componentes formados destacándose el componente 1 (Y1, Y3 y Y5) y el componente 2 (Y2, Y4 y Y6) (Figura 2).

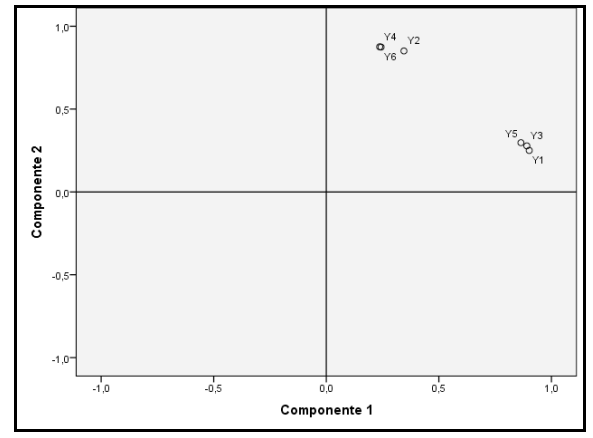

Figura 2. Gráfico de componentes principales rotado para las variables dependientes

Luego de haber verificado que las variables dependientes e independientes guardan una correlación bilateral significativa al nivel 0,01 o menor en el $100 \%$ de los casos (que corresponde a las 78 interacciones totales generadas), se concluye que la hipótesis principal propuesta se valida, lo cual se respalda que por debajo de este valor se encuentra el 100\% de los casos que evidencian dependencia entre las variables analizadas. Sin embargo, para 
las pruebas de hipótesis se consideraron los componentes extraídos luego del análisis factorial y las variables que mayor peso presentaron dentro de cada componente formado (Tabla 5).

Tabla 5. Resumen de las pruebas de hipótesis realizadas a componentes principales

\begin{tabular}{|c|c|c|c|c|}
\hline VI & VD & $\mathbf{R}$ & $\begin{array}{l}\text { SIG. (BILATE- } \\
\text { RAL) }\end{array}$ & DECISIÓN \\
\hline \multicolumn{5}{|c|}{ Variables dependientes } \\
\hline \multirow[t]{4}{*}{ X5 (CP1) } & Y1 (CP1) & 0,884 & 0,000 & $\begin{array}{ll}\text { Se rechaza } \\
\text { H0 }\end{array}$ \\
\hline & Y3 (CP1) & 0,887 & 0,000 & \\
\hline & Y5 (CP1) & 0,935 & 0,000 & \\
\hline & Y4 (CP2) & 0,604 & 0,000 & \\
\hline \multirow[t]{4}{*}{ X9 (CP1) } & Y1 (CP1) & 0,897 & 0,000 & $\begin{array}{ll}\text { Se rechaza } \\
\text { H0 }\end{array}$ \\
\hline & Y3 (CP1) & 0,901 & 0,000 & \\
\hline & Y5 (CP1) & 0,949 & 0,000 & \\
\hline & Y4 (CP2) & 0,615 & 0,000 & \\
\hline \multirow[t]{4}{*}{ X12 (CP2) } & $\mathrm{Y} 1(\mathrm{CP} 1)$ & 0,302 & 0,000 & $\begin{array}{ll}\text { Se rechaza } \\
\text { HO }\end{array}$ \\
\hline & Y3 (CP1) & 0,285 & 0,000 & \\
\hline & Y5 (CP1) & 0,319 & 0,000 & \\
\hline & Y4 (CP2) & 0,335 & 0,000 & \\
\hline \multirow[t]{4}{*}{ X2 (CP3) } & Y1 (CP1) & 0,580 & 0,000 & $\begin{array}{ll}\text { Se rechaza } \\
\text { H0 }\end{array}$ \\
\hline & Y3 (CP1) & 0,594 & 0,000 & \\
\hline & Y5 (CP1) & 0,620 & 0,000 & \\
\hline & Y4 (CP2) & 0,907 & 0,000 & \\
\hline
\end{tabular}

El resultado alcanzado en la prueba alfa de Cronbach $(0,877)$, realizada a partir de la información obtenida de la encuesta realizada al grupo de expertos, muestra la correlación que se presenta entre cada una de las preguntas realizadas las que fueron identificadas como variables independientes y dependientes; evidenciándose que el valor de la consistencia interna de la escala utilizada es alto, confirmándose estadísticamente que el instrumento aplicado para medir el impacto del modelo EMP-APP en el desarrollo socioeconómico de los cantones de la provincia de El Oro, presentan validez y confiabilidad. Según Celina y Campo, (2005) el valor mínimo que se acepta para el coeficiente alfa de Cronbach es 0,7.

\section{Propuesta. La asociatividad entre el sector público y privado.}

Actualmente la relación entre el sector público y privado se ha tornado muy dinámica y cada vez se presenta más apertura en la búsqueda de beneficios para la comunidad. El modelo plantea promover las APP institucionales para mejorar los indicadores socioeconómicos y como actores propone a las municipalidades, las IES y otras entidades como ministerios, ONG y fundaciones, para que juntos impulsen el desarrollo de proyectos de inversión en cada municipio. Bernal, Pumar, \& Zelada (2005), plantean un modelo en que la

\section{Cumbres}


municipalidad participa apoyando con la ejecución de obras relacionadas con la infraestructura pública y promueven la creación de condiciones para la implemetación de iniciativas que estimulan la participación del sector privado, sin formar parte de las empresas que puedan crearse. Otras oranizaciones como SIERRA EXPORTADORA (2011) y PROCASUR (2009) cuentan con modelos similares.

En el modelo se propone a las municipalidades como los protagonistas del desarrollo local con repercusiones a nivel provincial y nacional, a través de la creación del Departamento de Emprendimiento Municipal (DEM), encargado de promover la búsqueda de oportunidades de negocios mediante el establecimiento de alianzas estratégicas interinstitucionales con las IES y otras entidades para conformar Equipos Multidisciplinarios de Investigación y Desarrollo (EM/I+D), que se encargarán de realizar estudios que consideren aspectos económicos, sociales y ambientales para convertirlos en proyectos de inversión y luego en empresas productivas por sector de la economía denominadas Empresas Productivas Municipales en Alianza Público-Privada (EPM-APP-sector), constituidas por Unidades de Producción (UP), que operan de manera independiente dentro del sector en que se desarrolla. Además de Empresas Anexas que son elegidas para complementar las actividades de las EPM-APP.

La capitalización y fondeo de las EPM-APP, se realiza con instrumentos financieros como acciones y bonos negociados en el mercado de Valores del Ecuador, con límites de inversión para las acciones pero libre para inversiones en bonos con beneficio de cashback para los inversionistas usuarios. El personal para las EPM-APP, provendrá mayoritariamente (70\%) de las Universidades que conformaron los EMI+D, las municipalidades la diferencia (30\%). y se elegirán en base al modelo para selección y contratación de personal vigente para el sector público en Ecuador. Un porcentaje de las remuneraciones será cubierto en acciones hasta el límite establecido y luego se pagará en bonos. La Administración de las EPM-APP, estará a cargo de un Consejo Directivo con representación de todos los grupos que la conforman y se administrará bajo los principios de Gobierno Corporativo y Responsabilidad Social Empresarial. El modelo de gestión será el que se aplica para el sector público en Ecuador, además de auditorías externas (Figura 3).

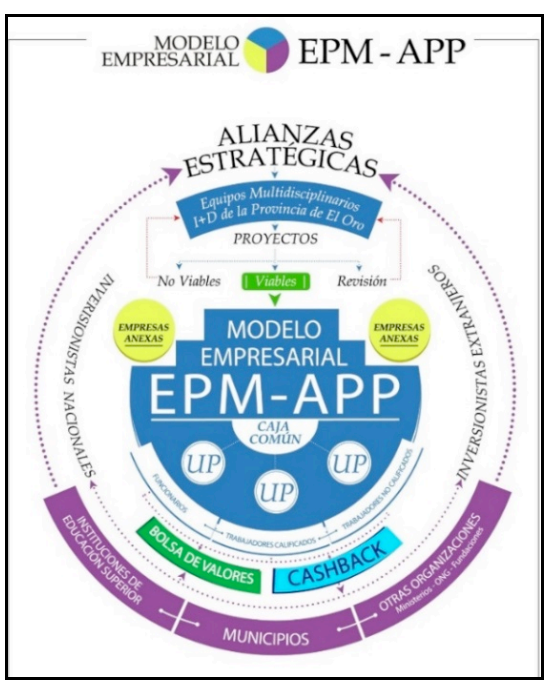

Figura 3. Modelo empresarial EPM-APP 


\section{CONCLUSIONES}

Se considera procedente el establecimiento de alianzas estratégicas interinstitucionales entre las municipalidades, universidades y otras entidades como las ONG, ministerios y fundaciones, debido a que permiten la creación de equipos multidisciplinarios de Investigación y Desarrollo (EM-I+D) que profundizan en las potencialidades de cada Cantón para la creación de las empresas con asociación de capitales público y privado que promuevan el desarrollo socioeconómico de la provincia de El Oro. El análisis estadístico otorga a la propuesta una consistencia interna alta (Índice Alfa de Cronbach de 0 ,877) y aceptación por parte de los ciudadanos orenses que fueron consultados, evidenciándose en las respuestas una contundente aprobación del modelo empresarial de APP que permita la creación de empresas con recursos provenientes del sector público y privado (EPM-APP) con la democratización de la propiedad, creadas por cada sector de la economía, para desarrollar los municipios de la provincia de El Oro.

\section{REFERENCIAS BIBLIOGRÁFICAS}

Almenara, J., Gonzalez, J., Garcia, C., \& Peña, P. (1998). ¿Qué es el análisis de componentes principales? Jano, 58-60.

Bernal, G., Pumar, A., \& Zelada, E. (2005). Metodología para la Implementación de un Municipio Productivo. Sucre, Bolivia: Editorial Tupac Katan.

Bolsa de valores de Quito. (20 de diciembre de 2016). www.bolsadequito.info/. Recuperado el 27 de diciembre de 2016, de www.bolsadequito.info/: http://www.bolsadequito.info/

Celina, H., \& Campo, A. (2005). Aproximación al uso del coeficiente alfa de Cronbach. Revista colombiana de Psiquiatría, XXXIV(004), 572-580.

Cronbach, L. J. (1951). Coefficient alpha and the internal structure of tests. Psychometrika, 16(1), 1-16.

González, Y. (2008). Instrumento Cuidado de comportamiento profesional: validez y confiabilidad", Chia, 8(2), 170-182.

H. Congreso Nacional. (2008). Ley de mercado de valores. Quito: Registro Oficial Suplemento 215 de 22-feb-2006.

Hair, J. F., Anderson, R. E., Tatham, R., \& Black, W. (1999). Análisis Multivariante. Quinta edición. Madrid: Prentice Hall Iberia.

Hernández, R., Fernández, C., \& Baptista, P. (2014). Metodología de la investigación (Sexta ed.). D.F. México: McGraw-Hill.

IBM. (2013). SPSS Statistics. Versión 22 para Windows. Valencia. España: Business Machines Corp.

INEC. (24 de enero de 2016). Instituto Nacional de Estadística y Censos. Obtenido de Instituto Nacional de Estadística y Censos: http://www.ecuadorencifras.gob.ec/

Jolliffe, I. (1986). Principal component analysis. Springler-Verlag.

Medina, A., Piloto, N., \& Nogueira, D. (2011). Estudio de la construcción de índices integrales para el apoyo al control de gestión empresarial. ENFO-

\section{Cumbres}


QUTE., 2, 1-39.

Moore, M. (2006). Crando valor público a través de asociaciones público-privadas. CLAD Reforma y Democracia(34), 1-22.

Neira, E. P. (2014). Machala, La ciudad bananera del mundo. Machala : Imprenta Tallpa Publicidad .

Pearson, K. (1900). On the criterion that a given system of deviations from the probable in. Philosophical Magazine , 5 th series (50), 157-175.

PROCASUR. (3 de Agosto de 2009). Ruta de aprendizaje. Obtenido de http:// www.procasur.org

Ríos, T. J., \& Torres, C. P. (2013). La tesis. Teoría y práctica. Lima: Cepredim, Primera edición. Universidad Nacional Mayor de San Marcos. ISBN. 978612-00-1192-8.

Rodríguez, G., Gil, J., \& García, E. (1996). Metodología de la Investigación Cualitativa. Málaga. España: Ediciones Aljibe.

Sepúlveda, B. (2014). Las asociaciones público privadas y el contrato de concesión: Regulación parcial en la Ley 1508 de 2012. Trabajo de grado. Tunja, Colombia.

SIERRA EXPORTADORA. (2011). Guía para el diseño e implementación del municipio productivo. Lima: Sierra Exportadora.

Sierra, E., \& Padilla, O. (1997). El Municipio como eje del desarrollo local (Vol. 1). Quito. Ecuador: Imprenta del Municipio Metroplitano de Quito.

Vasallo, J., \& Izquierdo, R. (2010). Infraestructura Pública y participación privada: conceptos y experiencias en América y España. Caracas: Venezuela. Corporación Andina de Fomento. 